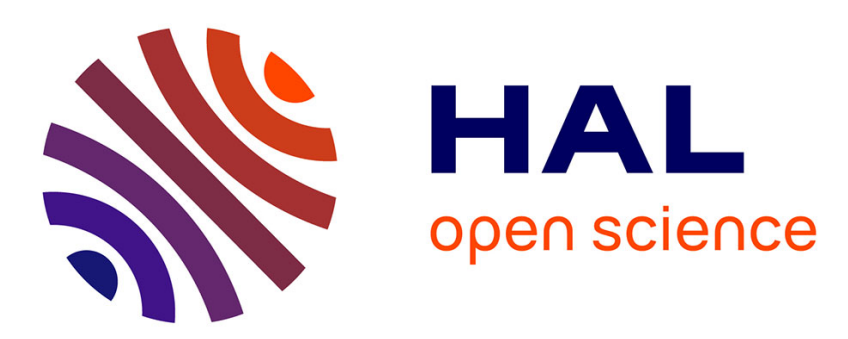

\title{
Application of a Multilayered Magnetostrictive Film to a Micromachined 2-D Optical Scanner
}

A. Debray, A. Ludwig, T. Bourouina, A. Asaoka, Nicolas Tiercelin, G. Reyne, T. Oki, E. Quandt, H. Muro, H. Fujita

\section{- To cite this version:}

A. Debray, A. Ludwig, T. Bourouina, A. Asaoka, Nicolas Tiercelin, et al.. Application of a Multilayered Magnetostrictive Film to a Micromachined 2-D Optical Scanner. Journal of Microelectromechanical Systems, 2004, 13 (2), pp.264-271. 10.1109/JMEMS.2004.825534 . hal-02973238

\section{HAL Id: hal-02973238 \\ https://hal.science/hal-02973238}

Submitted on 21 Oct 2020

HAL is a multi-disciplinary open access archive for the deposit and dissemination of scientific research documents, whether they are published or not. The documents may come from teaching and research institutions in France or abroad, or from public or private research centers.
L'archive ouverte pluridisciplinaire HAL, est destinée au dépôt et à la diffusion de documents scientifiques de niveau recherche, publiés ou non, émanant des établissements d'enseignement et de recherche français ou étrangers, des laboratoires publics ou privés. 


\title{
Application of a multilayered magnetostrictive film to a micromachined 2-D optical scanner
}

\author{
A. Debray ${ }^{1}$; A. Ludwig ${ }^{2}$; T. Bourouina ${ }^{1} ;$ A. Asaoka ${ }^{3}$; N. Tiercelin ${ }^{1} ;$ G. Reyne ${ }^{1}$; T. Oki ${ }^{1} ;$ E. \\ Quandt $^{2} ;$ H. Muro ${ }^{3} ;$ H. Fujita ${ }^{1}$ \\ 1- LIMMS/CNRS-IIS, University of Tokyo, Tokyo, Japan \\ 2- Center of Advanced European Studies and Research, CAESAR, Bonn, Germany \\ 3- Nissan Motor Company Limited, Yokosuka, Japan
}

\begin{abstract}
:
A novel two-dimensional (2-D) optical scanner has been designed, manufactured and characterized. This scanner features a large mirror $(8 / \mathrm{spl}$ times $/ 6 \mathrm{~mm})$ and is therefore suitable for industrial applications where cheap optical sources and lenses are requested. This scanner uses a multilayer film for its actuation. This film is well known for its high magnetostriction. The mechanical design has been optimized using conventional mechanical considerations as well as finite-element simulations. The device has been characterized in two configurations. Depending on the direction of the applied magnetic field, the magnetostrictive properties of the active film or the electromagnetic force are selectively used. Using this last, total optical deflection angles of 32/spl deg/ and 11/spl deg/ for an applied magnetic field of $0.3 \mathrm{mT}$ are obtained. The ratio of the corresponding resonant frequencies is around 4.5, allowing a nice scanning pattern. Compared to our previous prototype on the same project, the mechanicalmagnetic sensitivity has been improved by about a factor 24 when the magnetostriction is used, and by about a factor 75 when the electromagnetic force is used.
\end{abstract}

Published in: Journal of Microelectromechanical Systems (Volume: 13, Issue: 2, April 2004)

\section{Introduction}

The most widely used optical scanners today are polygon mirrors and galvanometric mirrors. Although research is continuing in these fields nowadays [1], research on optical scanners made with MEMS technology started at the beginning of the 1980s [2]. The applications for optical scanners are numerous and include display, barcode reading, printers, radar systems, and optical communication systems. Because of this diversity of applications for optical scanners, designs and actuation mechanisms are also numerous. Electrostatic [3], thermal [4], piezoelectric [5], electromagnetic [6] and magnetostrictive [7] scanners have been reported in the literature. They also include one-dimensional (1-D) and two-dimensional (2-D) scanners. However, most optical scanners made using MEMS technology and reported previously possess a small mirror (area is usually around $0.25 \mathrm{~mm}^{2}$ ). This size of mirror is unsuitable if simple optical elements as well as cheap laser sources are to be used, the size of the spot being rather $5 \mathrm{~mm}$ in diameter. It is only recently that optical scanners with large mirror area and made using MEMS technology have been reported [8] [9] [10].

The device presented in this article is included in this last category of devices where the size of the mirror is several $\mathrm{mm} 2$ and is the continuation of the work reported in [10]. Previous studies showed the possibility of using magnetostrictive actuation to fabricate 2-D optical scanners made by MEMS technology [7], [11]. More recent studies showed that this principle could be 
extended to make a 2-D scanner with a large mirror, good optical performances and including piezoresistive gauges to perform real-time position detection [10]. However, the deflection angles of this scanner appeared to be too small for the application. In order to over come this drawback, a new generation of scanner has been developed.

\section{Requirements}

The requirements for this device are the following:

- the scanner is a 2-D scanner;

- the mirror size is $6 \times 8 \mathrm{~mm} 2$;

- the peak-peak optical deflection angles are $40^{\circ}$ and $10^{\circ}$ in the two scanning directions, respectively;

- the ratio between the resonance frequencies is more than 5;

- the lowest resonance frequency is around $100 \mathrm{~Hz}$.

The size of the mirror is imposed by the desire to use a cheap laser source which spot size is 5 $\mathrm{mm}$ in diameter and simple optics. The deflection angles are specified by the application. The ratio between the resonance frequencies is to obtain a nice scanning pattern. Finally, the required value for the lowest resonance frequency is imposed by the electronic treatment of the signal.

Potential applications for such a device are related to objects detection in a given threedimensional (3-D) space.

\section{Design}

A scheme of the scanner with the used dimensions is presented in Fig. 1. It consists in a large mirror, a frame and four beams. The thickness of the mirror and of the frame is the same as the one of the wafer and therefore cannot be deformed during operation. The laser beam reflects directly on the polished silicon surface, which is sufficient for our study concentrated on the mechanical behavior of the device. The thickness of the beams is much smaller and therefore these lasts can be easily deformed. A magnetostrictive film is deposited on the backside of the whole device. This film also possesses a permanent and well-defined magnetization.

The working principle of this scanner is the following. Two mechanical resonant modes of the structure are used to provide a 2-D motion of the mirror. The actuation of the mirror will be performed using magnetostrictive force or electromagnetic torque.

In the case of magnetostrictive actuation, as the deformation of the magnetostrictive material is anisotropic, both the bending and torsion modes of the beams 1 (see Fig. 1) can be actuated depending on the frequency of the sinusoidal current applied to the driving coil. The torsion deformation of parts 1 is used for the maximum deformation angle in one direction. On the other hand, the bending deformation of parts 1 leads to a torsion deformation of parts 2 if the resonant frequency of the bending mode for the outer part (frame +parts 1) is the same as the resonant frequency of the torsion mode for the inner part (mirror and parts 2). Modal and harmonic simulations using ANSYS have been used in order to improve the design. For these simulations, the scanner was considered as an elastic shell which has both bending and membrane capabilities (ISHELL63 type in ANSYS). Each node has 6 degrees of freedom, three degrees of translation and three degrees of rotation. The mesh is automatically regenerated by 
the software using quadrilateral-shaped elements which characteristic size is $500 \mu \mathrm{m}$. The modal analysis is made using the subspace iteration method. The first 10 modes are calculated but only the two first are used for the analysis. For the harmonic response analysis, the mode superposition method is used. Fig. 2 shows the enhanced deformation obtained by simulation because of this principle where a bending torque has been applied on one of the beam 1, leading to higher rotation of the mirror, the beams 2 being then deformed in torsion.

It is also possible to use the magnetic interaction between the film magnetization and an applied external field. When the magnetization is in the plane, the application of a perpendicular magnetic field will lead to the generation of a torque, actuating the mirror. As we need rotations around two axes, we should set the magnetic moments in any privileged direction depending on the desired ratio for both deflection amplitudes. For instance, in the case of balanced torques, the magnetic moments of the material should remain in the $45^{\circ}$ direction. In our device, the magnetic moments of the material were chosen to be at $68^{\circ}$ from the $X$ axis of Fig. 1 in order to balance the torques in the case of magnetostrictive actuation (see [10] for details).

As of today, the best magnetostrictive layers are artificially nanostructured materials. Stacking nanometric layers of giant magnetostrictive Terfenol ( $\mathrm{TbFe} 2)$ and high magnetization $\mathrm{FeCo}$ allows the mixing of their properties due to the magnetic coupling between the layers [12]-[13] [14]. These layers are also suitable for the magnetic actuation. When depositing the film under a bias field, a magnetization as high as one Tesla can be obtained, as well as a well-defined inplane magnetic anisotropy in a chosen direction and a coercive field higher than the excitation field, in order to avoid the continuous switching of the magnetic moments during an alternative excitation. The use of $\mathrm{FeCo}$ in the layer provides the saturation magnetization, whereas $\mathrm{TbFe}$ contributes to the generation of a strongly privileged direction for the magnetic moments.

The shape of the cross section of the bars has also been optimized regarding their torsional stiffness and stress. The torsional stiffness and the maximum shear stress of a bar according to the shape of its cross section and its dimension when it is loaded in torsion can be found in mechanical engineering literature [15]. In the following discussion, we consider a bar of uniform cross-section. Torques are applied at the extremities of the bars and the deformation is supposed to be elastic. The torque $T$ to twist the bar of an angle $\theta$ is given by the following formula: $\quad T=(\theta / l) K G \quad(1)$

where $l$ is the length of the bar, $G$ the modulus of rigidity of the material ( $G \approx 69 \mathrm{GPa}$ for Silicon) and $K$ the torsional stiffness given in $\mathrm{m} 4$. It is obvious from this formula that for a given torque, the twisting angle will be more important if the bar is longer. If the cross section of the bar is considered to be a rectangle which size is $2 a$ by $2 b$, it is possible to calculate its torsional stiffness depending on the aspect ratio of the rectangle using formula (2)

$$
K=a b^{3}\left[\frac{16}{3}-3.36 \frac{b}{a}\left(1-\frac{b^{4}}{12 a^{4}}\right)\right]
$$

The result of the calculation, performed with on a constant cross-section area of 1 square meter, is presented in Fig. 3. It can be seen that the torsional stiffness rapidly decreases as the aspect ratio increases. In our design, the aspect ratio of the bars was around 133.

The maximum shear stress in the bar is related to the applied torque by the following formula: 
where $\alpha$ is a coefficient depending on the shape of the cross section of the bar and its cross sectional dimensions (formula can be found in [15]). Replacing $T$ by its value according to (1), the maximum shear stress is then given by

$$
\operatorname{Max} \tau=\alpha(\theta / l) K G
$$

For a given length $l$, rigidity $G$, and twisting angle $\theta$, the maximum shear stress is then depending on the cross-sectional shape according to the factor $\alpha K$. Fig. 4. shows the dependence of the maximum shear stress on the aspect ratio of a rectangular bar, again with a constant cross-section area of $1 \mathrm{~m} 2$. It can be seen that the maximum shear stress rapidly decreases as the aspect ratio increases.

From these calculations, we can see that when the aspect ratio of the beams are high, the stiffness and the maximum shear stress of the beams are reduced, leading to improved deflection angles and less fragility.

It can be seen from (1) and (4) that another solution to reduce the stiffness and maximum shear stress of the beams would have been to increase their length $l$ rather than to increase their crosssectional aspect ratio. This solution has not been investigated in this study in order to keep the device more compact.

Several dimensions like the size of the frame and the length of the torsional bars have been adjusted using modal simulations with ANSYS in order to adjust the resonance frequencies according to the requirements.

\section{Process}

The process flow chart is presented in Fig. 5. It starts with $15-\mu \mathrm{m}$-thick SOI wafers. The shape of the scanners is patterned on the front side using deep RIE with a photoresist mask. The backside is then etched using deep RIE with an aluminum mask. The structures are released using BHF. Finally, the active material is sputtered everywhere on the backside. The thickness of the mirror and of the frame is supposed to be thick enough so they do not deform during operation. A magnetic field is applied during the deposition in order to induce an easy axis in the thin film. The mechanical stress in the torsional spring is small enough so that no further annealing is needed.

Besides this process, some wafers had their backside thinned in order to have some devices with a lighter frame and mirror, and therefore higher resonant frequencies. The thinning of the backside has been performed by $\mathrm{KOH}$, the front side being protected by silicon nitride that has been deposited by LPCVD. It took four and a half hour to etch approximately $300 \mu \mathrm{m}$ in a $30 \%$ $\mathrm{KOH}$ solution at $80 \circ \mathrm{C}$. Two hundreds nanometers of silicon nitride were sufficient to protect the front side during this etching. On a 3-inch wafer, the difference of etching speed between two positions is not greater than $10 \%$. The silicon nitride is then removed using pure HF and the process described in the previous paragraph is applied to these thinned SOI wafers. 
The thin film consists of a magnetron-sputtered TbFe-CoFe multilayer [13]. Each layer has a thickness of about $10 \mathrm{~nm}$ and the total thickness of the film is around $4 \mu \mathrm{m}$. This layer was sputtered under a magnetic bias field in the $68^{\circ}$ direction from the $X$-axis in order to balance the bending and twisting deformation of the hinges in the magnetostrictive case.

\section{Characterization}

Two different configurations have been used in order to characterize the magneto-mechanical properties of the scanners, using the magnetostrictive force or the electromagnetic torque.

Magnetostrictive actuation is performed via the rotation of the magnetic moments in the plane of the active layer. The magnetostrictive deformation is mainly generated in the bars. Therefore, for this method, we set up an "in-plane" configuration: In this configuration, the magnetic field is in the plane of the thin film and localized on one of the external torsion bars. A scheme of this configuration as well as a picture of the experimental set-up is presented in Fig. 6. This magnetic field is obtained using two coils placed side-by-side and wound in opposite direction as described on the schematic. The diameter of the coils is around $1 \mathrm{~cm}$. They are driven by a sinusoidal current. The magnetic field is proportional to the current in the coils, and is calibrated using a Hall effect probe. At around $37 \mathrm{~Hz}$, the first mechanical mode of the structure is excited. In this mode, the external beams are deformed in torsion. For a magnetic field of $0.3 \mathrm{mT}$, the total optical deflection angle is $8.5^{\circ}$. Around $200 \mathrm{~Hz}$, the second mode of the structure is excited. In this mode, the external beams are deformed in bending, whereas the inner beams are deformed in torsion. This inner torsional deformation induced by the outer bending deformation is therefore observed as it was predicted during the design presented in Section III. For a magnetic field of $0.3 \mathrm{mT}$, the total optical deflection angle is $2^{\circ}$.

We also investigated the electromagnetic actuation. As seen in Section III, this requires the application of an out of plane external magnetic field, hence the "out-of-plane" configuration. In this configuration, the magnetic field is applied on the entire sample and is perpendicular to the thin film. This magnetic field is produced by one coil, which diameter is $7 \mathrm{~cm}$ and in which the sample is placed. A scheme of this configuration as well as a picture of the experimental set-up is presented in Fig. 7. As previously, this coil is driven by a sinusoidal current. The two first modes of the mechanical structure can be excited. For a magnetic field of $0.3 \mathrm{mT}$, the first mode is at $52 \mathrm{~Hz}$, the total optical deflection being $32^{\circ}$. For the same magnetic field of $0.3 \mathrm{mT}$, the second mode is at $235 \mathrm{~Hz}$, the total optical deflection being $11^{\circ}$. The frequency responses for these first two modes for a magnetic field of $0.3 \mathrm{mT}$ are presented in Fig. 8. The behavior of these scanners is seen to be nonlinear with a hard spring effect. When the coil is driven by the addition of two sinusoidal currents at $50 \mathrm{~Hz}$ and $230 \mathrm{~Hz}$ respectively, and that the magnetic field produced is $0.8 \mathrm{mT}$, the two first modes of the structure are excited simultaneously. The total optical deflection produced by the first mode is of $35^{\circ}$, and the total optical deflection produced by the second mode is of $6^{\circ}$. Another result concerns the cross talk between the first two modes. With a sinusoidal excitation of $0.5 \mathrm{mT}$ at $42 \mathrm{~Hz}$, the first mode produces a total optical deflection of $17^{\circ}$, and the second mode, which resonant frequency has been seen to be above $200 \mathrm{~Hz}$, produces a total optical deflection of $1^{\circ}$.

When the frame and the mirror are thinner, the same behaviors are observed, but the resonant frequencies are higher. For an exciting magnetic field of $0.13 \mathrm{mT}$, the first mode resonates at $55 \mathrm{~Hz}$, and the second mode at $335 \mathrm{~Hz}$. However, we have to note that the differences in the resonant frequency for each mode compared when using the magnetostrictive force or the 
electromagnetic torque are due to the hard spring effect which implies that the resonant frequency of each modes increases when the deformation amplitude also increases.

\section{Discussion}

From the characterization, we can see that using the electromagnetic actuation with a magnetic field of $0.8 \mathrm{mT}$ almost fulfills the requirements for the deflection angles, the ratio between the resonant frequencies and the values of these lasts. Moreover, it is important to compare these results with the one obtained with the previous design presented in [10]. This device was shaped like a cantilever and will be referred to as the cantilever-like scanner. One thick part (more than $200 \mu \mathrm{m}$ ) was supporting the mirror, while a thin part (from 10 to $20 \mu \mathrm{m}$ depending on the samples), clamped to the wafer and the mirror, could be easily deformed. The same active material as the one used here was sputtered on the backside of the whole samples with the same thickness. The size of the mirror was the same as the one of the current device. The important parameter for comparison is the mechanic-magnetic sensitivity, which is defined as the ratio of total optical deflection angle to the applied magnetic field. When actuated using the magnetostrictive force, the cantilever-like scanner showed a sensibility of $1.2 \mathrm{deg} / \mathrm{mT}$ and 0.3 $\mathrm{deg} / \mathrm{mT}$ for the torsion and bending deformation of the thin part, respectively. When the magnetostrictive force is used to actuate the scanner presented in this article, the sensibility is $28,3 \mathrm{deg} / \mathrm{mT}$ and $6,6 \mathrm{deg} / \mathrm{mT}$ for the torsion and bending deformation of the outer beam, respectively. The improvement from one device to another is very important. The measurements for the last scanners have been made separately by the three collaborating institutions involved in the project. The measurements for both designs have been made on several runs, therefore confirming the repeatability of the fabrication and of the results.

This improvement can be explained by three factors. First, the mechanical design of the structures has been changed. The beams have been designed longer and less wide in order to improve the torsion angle for a given torque. Second, the target for the Terfenol ( $\mathrm{TbFe} 2)$ has been changed between the realizations of the two types of devices. The manufacturer of this new target was a different one than the one for the first target. Finally, some changes in the process could have changed the properties of the deposited active film. The former realized cantilever-like scanners were including piezoresistive gauges. In order to protect these gauges, a thick negative photoresist (OMR $300 \mathrm{CP}$ ) was deposited on these lasts. After the sputtering of the active film, this photoresist was removed either by $\mathrm{O}_{2}$ ashing in RIE or by UV-ozone stripping. The temperature of the $\mathrm{O}_{2}$ ashing in RIE was unknown, while the temperature of the UV-ozone stripping was set to $150 \circ \mathrm{C}$. These operations could last up to 2 or 3 hours depending on the samples. It is suspected, although not confirmed, that this step dedicated to remove the OMR photoresist could have damaged the properties of the active film, either by disorientating the magnetic moments of the material, either by mixing the nanolayer components by diffusion, or by both. A new process avoiding this last step is under investigation at our institute.

Another possible comparison is between the magnetostrictive actuation and the electromagnetic force. In the case of magnetostrictive actuation, the sensibility is $28,3 \mathrm{deg} / \mathrm{mT}$ and $6,6 \mathrm{deg} / \mathrm{mT}$. In the case of the electromagnetic force, the sensibility is $70 \mathrm{deg} / \mathrm{mT}-24 \mathrm{deg} / \mathrm{mT}$. Although this material has been optimized in order to be highly magnetostrictive, the use of electromagnetic force for this device greatly improves the sensibility compared to the use of the magnetostrictive actuation. This is mainly explained by the size of the device. The magnetostrictive torque acts on the surface of the beam where the magnetic film is applied, whereas the electromagnetic force acts on the whole volume of the active material. In this particular device, the design 
allowed us to have a huge volume of active material compared to the surface where the magnetostrictive torque applies. This is due to our requirement of having a large mirror. Therefore, for other applications, size of device and designs, the magnetostrictive properties of this film can be preferred to its purely magnetic ones, especially if small volumes and large areas of active material have to be used. Moreover, the choice between magnetostrictive and electromagnetic force may be influenced by considerations on the packaging when the magnetic actuation has to be taken into consideration. Electroplated coils made by MEMS technology [16] or "mini" coils as the one used in millimeter size motors could be used for easy assembly and therefore may be low costs. The geometry of the device could influence the use of one or the other property of the film. Concerning our device, the magnetostrictive efficiency could be increased by exciting simultaneously the two outer beams referred to as 1 but fed with opposite driving currents, leading to a push-pull actuation.

\section{Conclusion}

A new 2-D optical scanner has been designed, manufactured and characterized. This scanner is intended for industrial applications and therefore features a large mirror. The use of MEMS techniques for its fabrication is intended to reduce to costs by the use of batch process. Its actuation is obtained by the use of a multilayer film that is well known for its optimized magnetostrictive properties. Design using conventional mechanical engineering knowledge as well as finite-element method calculations has been used in order to optimize the mechanical features of the device.

Two ways of actuation have been investigated. One uses the magnetostrictive properties of the active film whereas the other one uses the electromagnetic force. It turns out that this last is more suitable for this device, due to its particular geometry and its large size. The requirements concerning the resonant frequencies, frequencies ratio and deflection angles have been almost met. When compared to the previous prototype of the same project, it appears that the mechanical-magnetic sensitivity of the device has been greatly improved. This is due to the mechanical design optimization and modifications in the process.

Although the requirements have been almost fulfilled, it is expected that this device will be too fragile for several applications. Measurements concerning this feature are not available so far, but several samples have been broken during transportation from one institute to another, therefore delaying the project. It is believed that it is in this direction that improvements have two be made. Two different ways to achieve this goal are under investigation. The first approach is to use a purely magnetostrictive actuation whose sensitivity is greatly enhanced by a "field induced" magnetic instability [17]. A new design of cantilever based scanners with $50-\mu \mathrm{m}$ thick actuation parts, and therefore more robust, is currently under investigation. First results show large optical deflections despite no optimization. On the other hand, promising new materials with low young's modulus and high resilience such as the silicon polymer based PDMS could be used in association with silicon to provided at the same time robust mechanical properties, integrated electronics and batch fabrication process.

\section{Acknowledgements}

This project was led in the framework of LIMMS, Laboratory for Integrated MicroMechatronics Systems, located in Tokyo, a joint laboratory between the Science and Technology Department of Information and Communication of CNRS (CNRS-STIC, France) and the Institute of Industrial Science (IIS-the University of Tokyo). It was a collaborative work 
together with CAESAR (Center of Advanced European Studies and Research), Bonn, Germany, and the Nissan Motor Company, Ltd., and also in the framework of CIRMM (Center of International Research for Micro-Mechatronics). The authors would like to thank Prof. H. Hashiguchi from Kagawa University, Japan, and all the members of his laboratory for the help in the processing of some scanners as well as for useful advices.

\section{References}

1.M. Hafez, T. C. Sidler, R. P. Salathé, G. L. M. Jansen and J. C. Compter, "Design simulations and experimental investigations of a compact single mirror tip/tilt laser scanner", Mechatronics, vol. 10, pp. 741-760, 2000.

2.K. E. Petersen, "Silicon torsional scanning mirror", IBM J. Res. Develop., vol. 24, no. 5, pp. 631-637, 1980.

3.R. A. Conant, P. M. Hagelin, U. Krishnamoorthy, O. Slogaard, K. Y. Lau and R. S. Muller, "A raster-scanning full-motion video display using polysilicon micromachined mirrors", Proc. Transducers'99, pp. 376-379, 1999.

4.S. Schweizer, P. Cousseau, G. Lamel, S. Calmes and P. Renaud, "Two-Dimensional thermally actuated optical microscanner", Proc. Eurosensors XIII, pp. 29-32, 1999.

5.T. Kawabata, M. Ikeda, H. Goto, M. Matsumoto and T. Yada, "The 2-dimensional micro scanner integrated with PZT thin film actuator", Proc. Transducers'97, pp. 339-342, 1997. 6.N. Asada, H. Matsuki, K. Minami and M. Esashi, "Silicon micromachined galvano optical scanner", IEEE Trans. Magn., vol. 30, pp. 4647-4649, 1994.

7.A. Garnier, T. Bourouina, E. Orsier, T. Masuzawa, H. Fujita, T. Hiramoto, et al., "A fast simple and robust 2-D micro-optical scanner based on contactless magnetostrictive actuation", Proc. MEMS'2000, pp. 715-720, 2000.

8.H. Miyajima, N. Asaoka, M. Arima, Y. Minamoto, K. Murakami, K. Tokuda, et al., "A durable shock-resistance electromagnetic optical scanner with polyimide-based hinges", $J$. Microelectromech. Syst., vol. 10, pp. 418-423, 2001.

9.N. Asada, M. Takeuchi, V. Vaganov, N. Belov, S. in't Hout and I. Sluchak, "Silicon microoptical scanner", Sens. Actuators Phys. A, vol. 83, pp. 284-290, 2000.

10.T. Bourouina, E. Lebrasseur, G. Reyne, A. Debray, H. Fujita, A. Ludwig, et al., "Integration of two degree-of-freedom magnetostrictive actuation and piezoresistive detection: Application to a two-dimensional optical scanner", J. Microelectromech. Syst., vol. 11, pp. 355-361, 2002.

11.T. Bourouina, A. Garnier, H. Fujita, J.-C. Peuzin and T. Masuzawa, "Magneticallyinduced frequency shift in a magneto-mechanical bimorph resonator", Proc. SPIE, vol. 4175, pp. 180-191, 2000

12.E. Quandt, A. Ludwig, J. Betz, K. Mackay and D. Givord, "Giant magnetostrictive spring magnet type multilayers", J. Appl. Phys., vol. 81, no. 8, pp. 5420-5422, 1997.

13.E. Quandt and A. Ludwig, "Giant magnetostrictive multilayers", J. Appl. Phys., vol. 85, no. 8, pp. 6232-6237, 1999.

14.H. L. Gall, J. B. Youssef, F. Socha, N. Tiercelin, V. Preobrazhensky and P. Pernod, "Low field anisotropic magnetostriction of single domain exchangeCoupled ( $\mathrm{TbFe} / \mathrm{Fe})$ multilayers", J. App. Phys., vol. 87, no. 8, 2000.

15.W. C. Young, Roark's Formulas on Stress and Strain, New York:McGraw- Hill.

16.L. Houlet, P. Helin, T. Bourouina, G. Reyne, E. Dufour-Gergam and H. Fujita, "Movable vertical mirror arrays for optical micro-switch matrices and their electromagnetic actuation", IEEE J. Select. Topics Quantum Electron., vol. 8, no. 1, Jan./Feb. 2002. 
17.N. Tiercelin, J. B. Youssef, V. Preobrazhensky, P. Pernod and H. L. Gall, "Giant magnetostrictive superlattices: From spin reorientation transition to MEMS. Static and dynamical properties", J. Mag. Mag. Mat., vol. 249, no. 3, pp. 519-523, 2002.

\section{Figures}

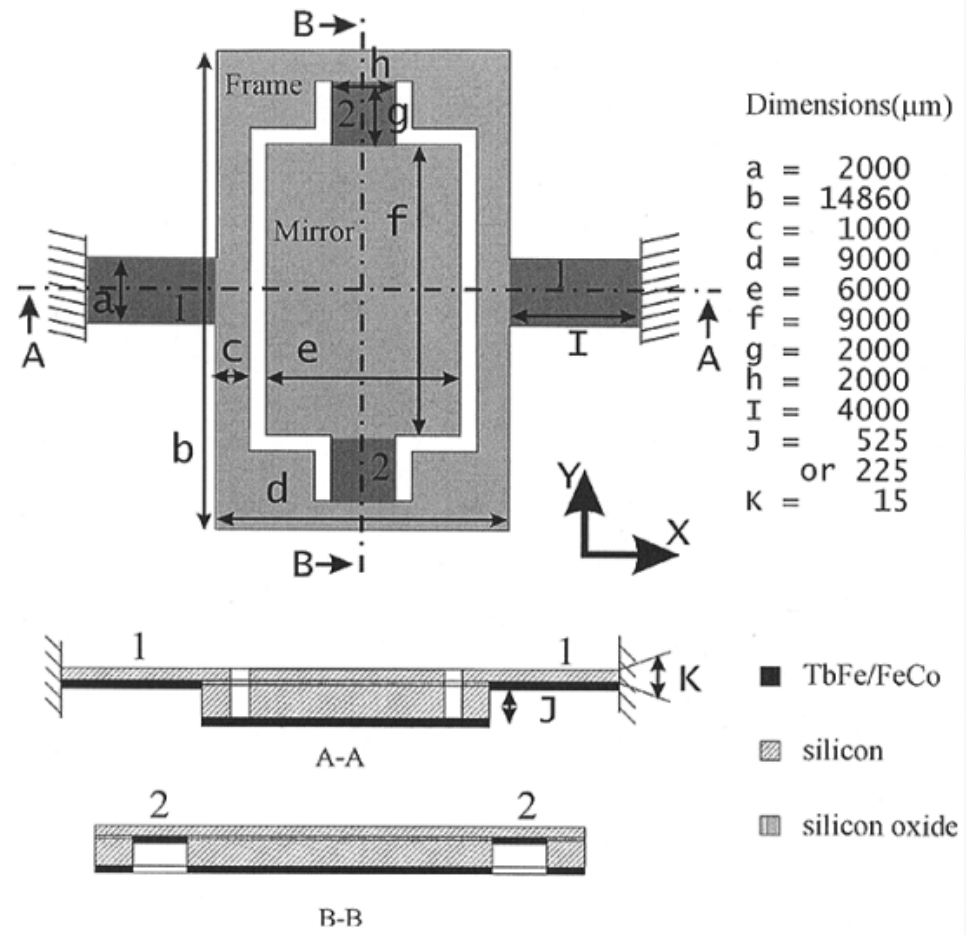

Fig. 1. Scheme of the 2-D optical scanner.

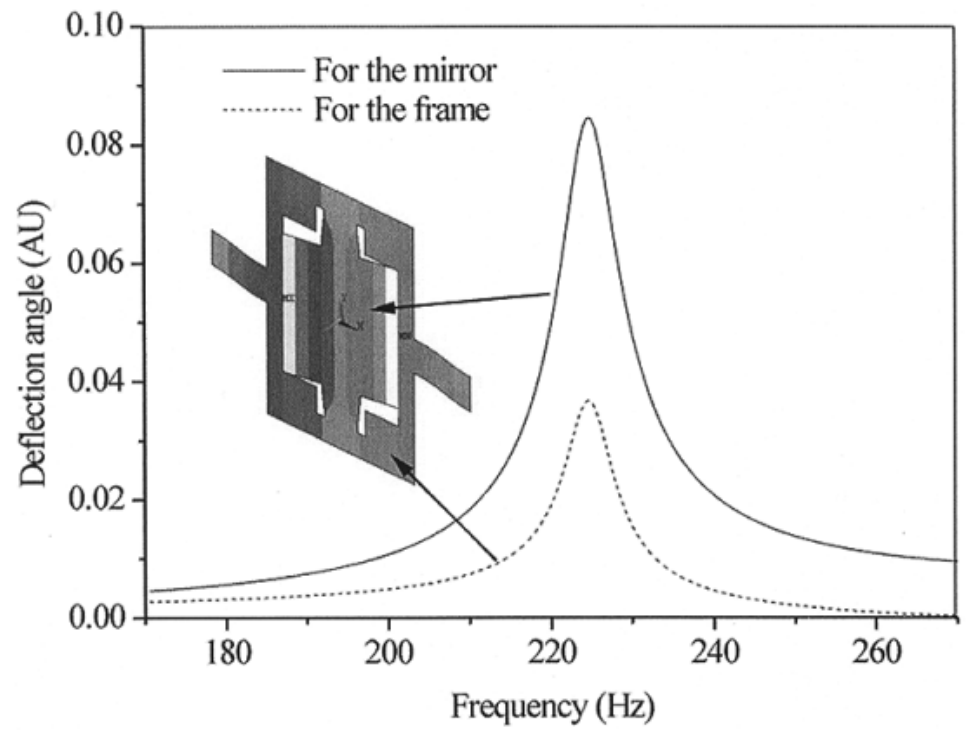

Fig. 2. Comparison of the angular deflection frequency responses of the mirror and of the frame when one of the two beams 1 (see Fig. 1) is excited in bending. 


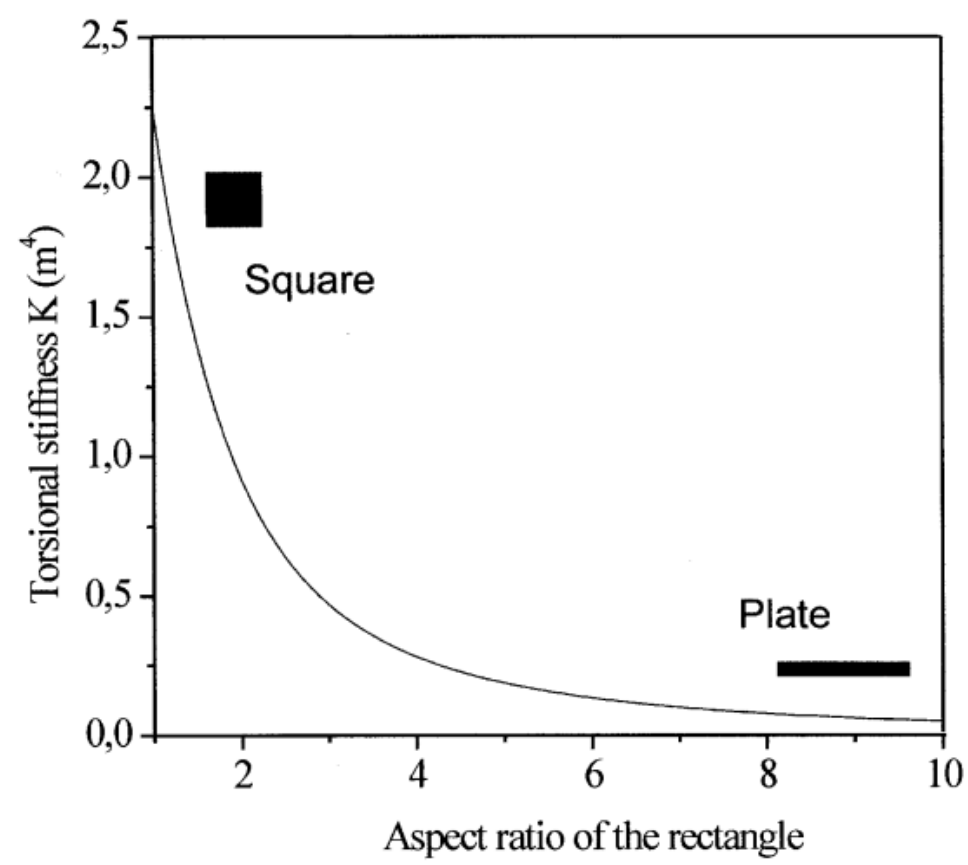

Fig. 3. Torsional stiffness of a rectangular bar deformed in torsion as a function of the aspect ratio of its cross-section, for a constant cross-section area of $1 \mathrm{~m}^{2}$. Aspect ratio of the final device is approximately 133 .

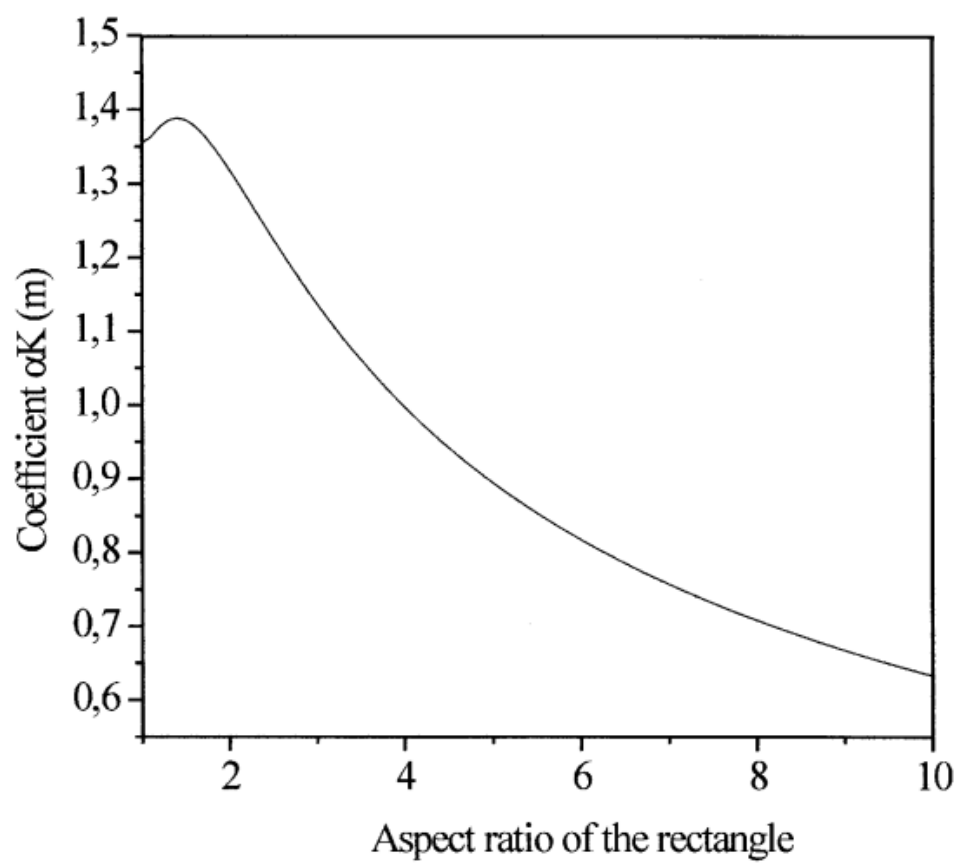

Fig. 4. Coefficient $\alpha K$, related to the maximum shear stress, of a rectangular bar deformed in torsion as a function of the aspect ratio of its cross-section, for a constant cross-section area of $1 \mathrm{~m}^{2}$. Aspect ratio of the final device is approximately 133 . 


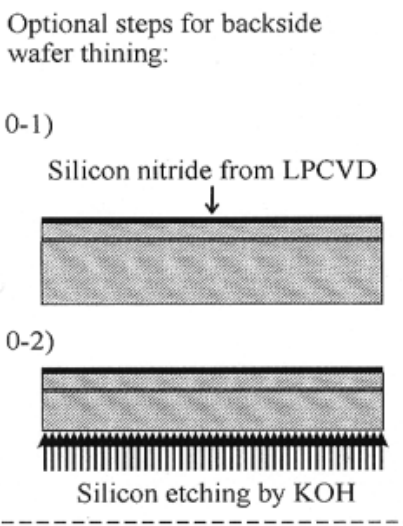

a)

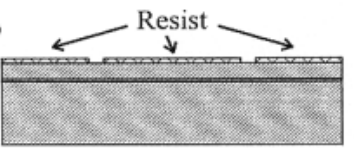

b)

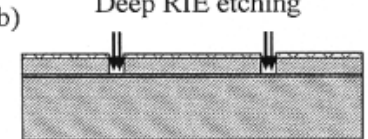

c)

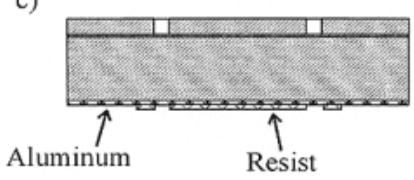

d)

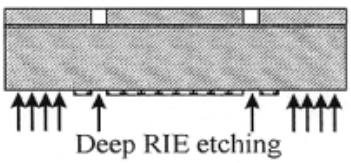

e)

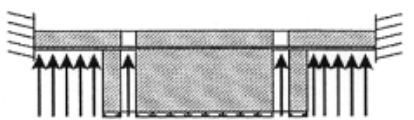

BHF wet etching of $\mathrm{SiO}$

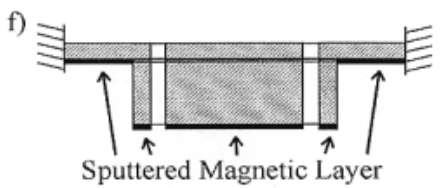

Fig. 5. Process flow chart for the A-A cross section as specified in Fig. 1.
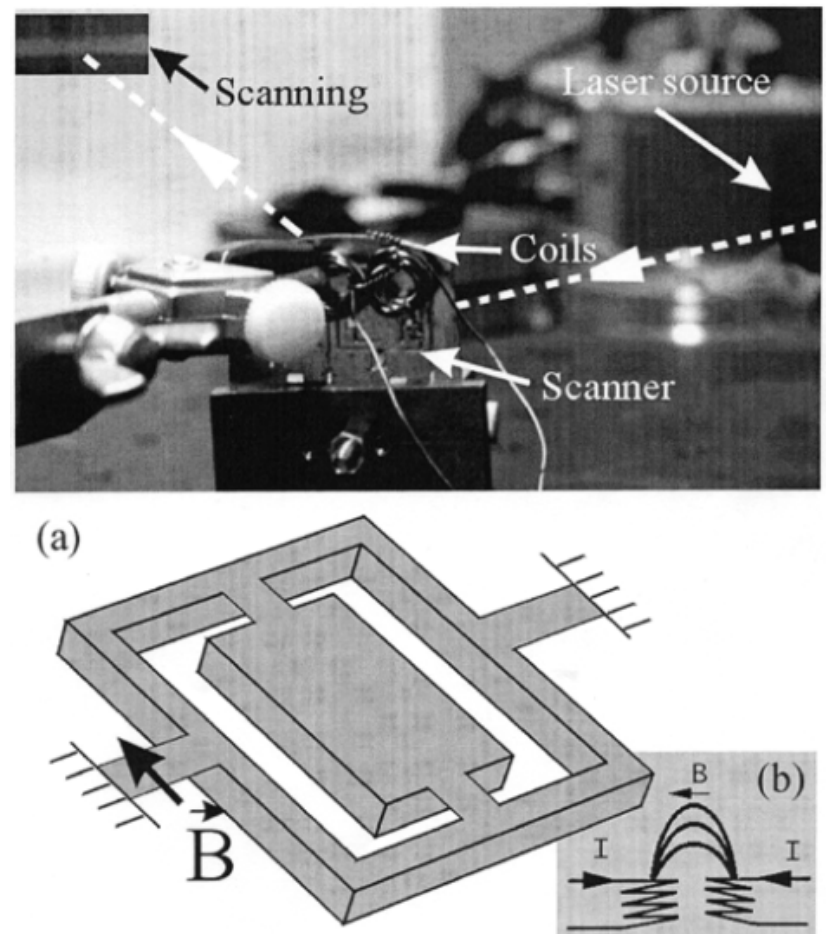

Fig. 6. The in-plane configuration: bottom: scheme showing (a) the field being applied to one of the hinges and (b) the coil configuration used to obtain the in-plane field-top: picture of the configuration showing the two magnetic coils in front of the 2-D optical scanner. The contrast of the scanned area has been artificially increased using a photo editor tool in order to improve the readability. 

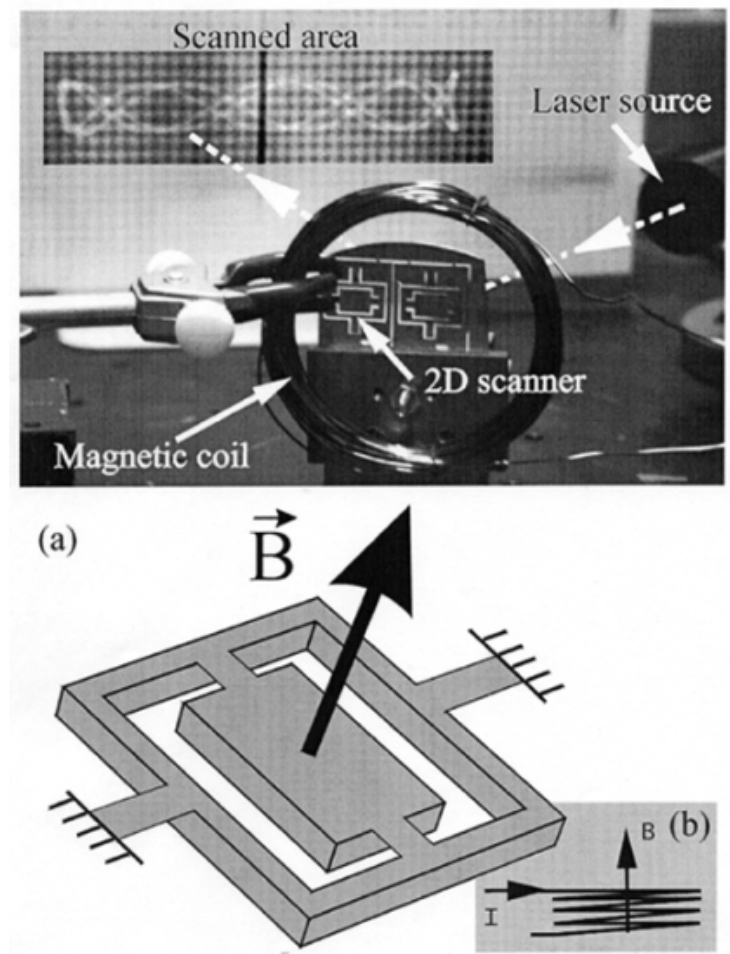

Fig. 7. The out-of-plane configuration: bottom: showing (a) the field being applied to perpendicularly to the mirror and (b) the coil configuration for the out of plane field-top: picture of the configuration showing the magnetic coil in front of two 2-D optical scanners. On the right we can see the laser source and on the back the scanned area. The contrast of the scanned area has been artificially increased using a photo editor tool in order to improve the readability.
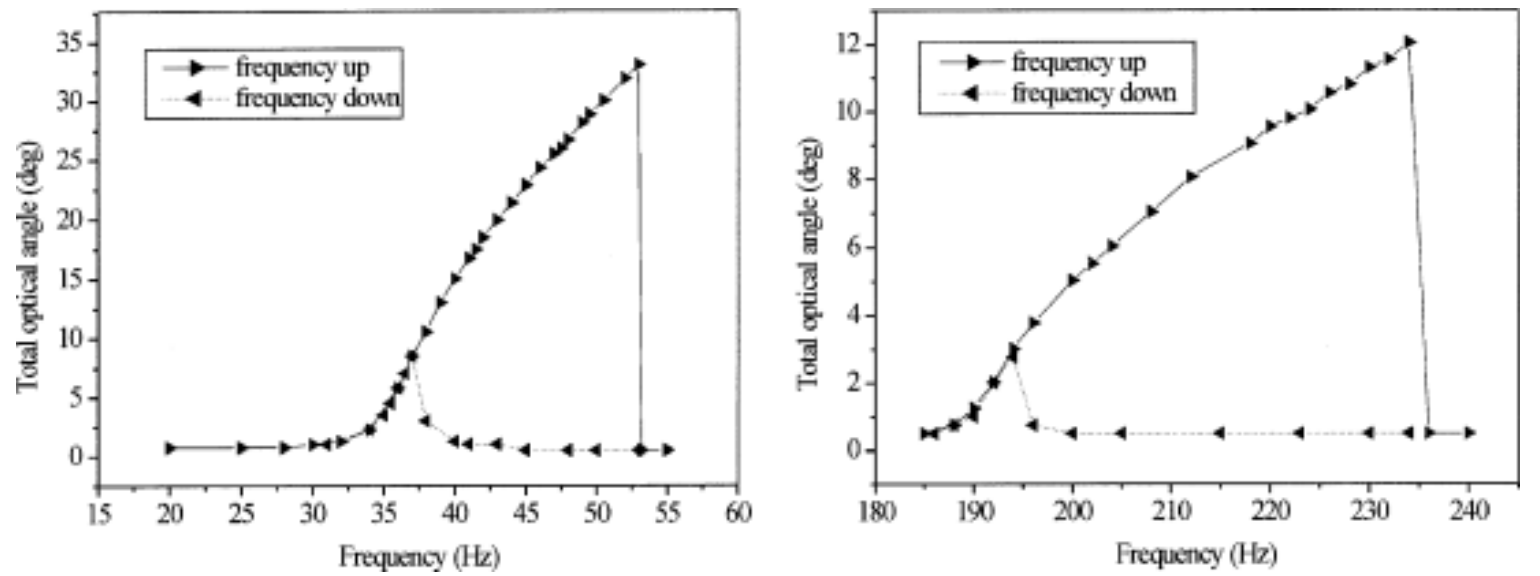

Fig. 8. The total optical deflection angle frequency response in the out-of-plane configuration for a magnetic field of $0.3 \mathrm{mT}$. Left: The outer beams being deformed in torsion. Right: The inner beams being deformed in torsion. 